EPJ Web of Conferences 41, 02023 (2013)

DOI: $10.1051 /$ epjconf/20134102023

(C) Owned by the authors, published by EDP Sciences, 2013

\title{
Time-resolved spectroscopy of doubly-excited states in helium
}

\author{
Christian Ott ${ }^{1,2}$, Andreas Kaldun ${ }^{1,2}$, Philipp Raith ${ }^{1,2}$, Kristina Meyer ${ }^{1,2}$, Martin Laux ${ }^{1,2}$, Yizhu \\ Zhang $^{1,2}$, Steffen Hagstotz ${ }^{1,2}$, Thomas Ding ${ }^{1,2}$, Robert Heck ${ }^{1,2}$, and Thomas Pfeifer ${ }^{1,2}$ \\ ${ }^{1}$ Max-Planck Institut für Kernphysik, Saupfercheckweg 1, 69117 Heidelberg, Germany \\ ${ }^{2}$ Center for Quantum Dynamics, Ruprecht-Karls-Universität Heidelberg, 69120 Heidelberg, \\ Germany
}

\begin{abstract}
A two-electron wave packet among the lowest-lying doubly-excited states in helium is experimentally observed. It creates a 1-femtosecond modulation in the transient-absorption signal modified by a time-delayed coupling laser.
\end{abstract}

\section{Introduction and Motivation}

Electron wave packets are the quantum description of electrons in motion. Whenever more than one electronic state is coherently populated, a dynamical wave packet is formed. The characteristic time period of wave-packet evolution $\Delta t$ corresponds to the inverse level spacing $h / \Delta E$. Thus, low-lying electronic states in atoms and molecules with a typical level spacing on the order of $\mathrm{eV}$ correspond to the temporal regime of few femtoseconds to hundreds of attoseconds.

Wave packets formed by single electrons on attosecond time scales have recently been observed in helium [1,2]. For the case of two electrons, dynamical wave packets have thus far only been predicted theoretically [3]. Here, we measure a two-electron wave packet in helium via the coherent laser-induced coupling of low-lying autoionizing states [4]. Our experimental scheme combines attosecond transient-absorption spectroscopy [5] with optical detection at high spectral resolution ( 20 meV@60 eV), which enables us to observe time-delay dependent changes on the spectrally narrow Fano absorption lines to evidence the two-electron wave packet.

\section{Experimental Scheme and Setup}

We use $\sim 7 \mathrm{fs}$ visible (VIS, centered around $\sim 730 \mathrm{~nm}$ ) laser pulses to generate spectrally continuous attosecond-pulsed soft-X-ray (SXR) radiation around the 60-70 eV energy range. The SXR pulses and the copropagating VIS driver light are spatially and temporally separated and both refocused into a 100 mbar helium cell. The transmitted SXR light is spectrally resolved with a flat-field spectrograph.

The targeted energy levels belong to the $N=2$ doubly-excited states series in helium, ranging between 60.15 and $65.40 \mathrm{eV}$. The SXR light coherently excites the dipole-allowed $2 \mathrm{~s} 2 \mathrm{p}\left({ }^{1} \mathrm{P}^{0}\right)$ and $\mathrm{sp}_{2,3+}\left({ }^{1} \mathrm{P}^{\mathrm{o}}\right)$ two-electron states from the $1 \mathrm{~s}^{2}\left({ }^{1} \mathrm{~S}^{\mathrm{e}}\right)$ ground state. Within their autoionization lifetime (17 fs for the fastest autoionizing $2 \mathrm{~s} 2 \mathrm{p}$ state [6]) these states are coherently coupled with a timedelayed VIS laser pulse by a two-photon transition via the intermediate $2 \mathrm{p}^{2}\left({ }^{1} \mathrm{~S}^{\mathrm{e}}\right)$ state (Figure 1). The energy-resolved dipole emission (with respect to the ground state) from these states carries a

This is an Open Access article distributed under the terms of the Creative Commons Attribution License 2.0, which permits unrestricted use, distribution, and reproduction in any medium, provided the original work is properly cited. 
time-delay-dependent modulation due to the interference between the direct transition pathway (SXR light only) and the time-delayed coupling between the states (both SXR and VIS light). This VIScoupled dipole emission is imprinted onto the transmitted and spectrally resolved SXR radiation which serves as the probe of the system. The here described experimental approach can thus be understood more easily as a transient-coupling scheme.

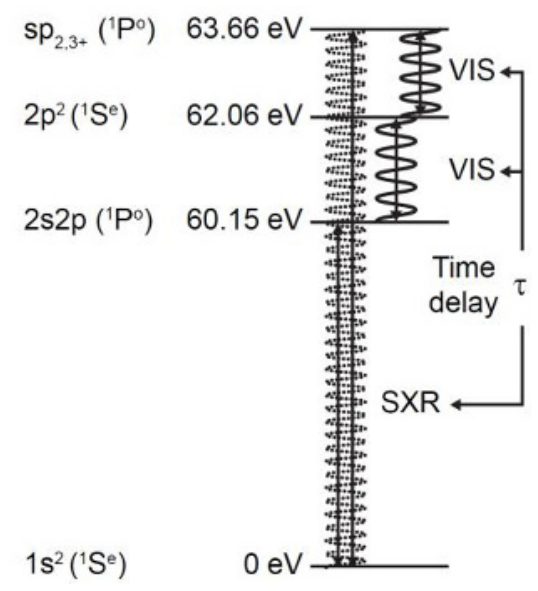

Fig. 1. Level scheme of the helium doubly-excited states contributing to the observed twoelectron wave packet. The SXR light excites the dipole-allowed (with respect to the $1 \mathrm{~s}^{2}$ ground state) states $2 \mathrm{~s} 2 \mathrm{p}$ and $\mathrm{sp}_{2,3+}$. The time-delayed $(\tau)$ VIS light couples these states via the intermediate dipole-allowed $2 \mathrm{p}^{2}$ state. The configuration-interaction coupling of these autoionizing states with single-ionization continua is not shown here for simplification, but accounted for in our model simulation.

\section{Experimental Results}

The SXR absorption measured after passage through the laser-dressed helium target is shown in Figure 2a,b for various time-delays between the SXR and VIS pulses. While for a negative time delay (VIS pulse arriving first) the well-known Fano absorption line shape is resolved, its shape changes significantly for positive time delays. In addition to a shifting and splitting across the lowest $2 \mathrm{~s} 2 \mathrm{p}$ resonance, as previously observed [6,7], a rapid time-delay dependent modulation in the absorption signal is evident across both the $2 \mathrm{~s} 2 \mathrm{p}$ as well as the $\mathrm{sp}_{2,3+}$ resonance. The modulation period is $(1.23 \pm 0.07) \mathrm{fs}$ and agrees with the inverse level spacing between the $2 \mathrm{~s} 2 \mathrm{p}$ and $\mathrm{sp}_{2,3+}$ states (energy difference $3.51 \mathrm{eV}$ corresponds to $1.18 \mathrm{fs}$ beating period). We reproduce the observed modulation and spectral changes via the theoretical modelling of the three $2 \mathrm{~s} 2 \mathrm{p}, 2 \mathrm{p}^{2}$ and $\mathrm{sp}_{2,3+}$ doubly-excited states including their laser-induced coupling (Figure 2c).

\section{Conclusion}

We observed a two-electron wave packet in helium, oscillating on a 1-fs timescale, on low-lying (low principal quantum number $n$ ) quantum states. These strongly correlated states are particularly important for chemistry, as molecular bonds are based on such near-valence-shell orbitals typically occupied by two electrons. Being able to laser-couple these states highlights a route to steer correlated two-electron motion within chemically relevant transition states, to ultimately control chemical reactions on their natural electronic time scale. 

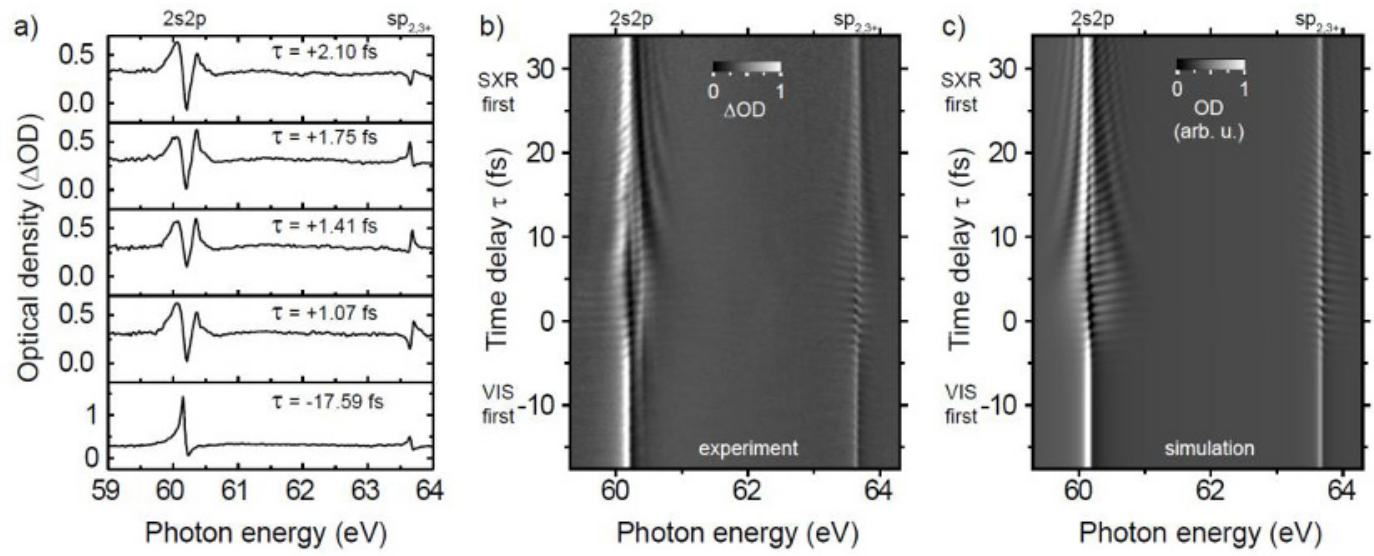

Fig. 2. Experimental evidence of the two-electron wave-packet beating. a) Transientabsorption profiles of the 2s2p (@60.15 eV) and $\mathrm{sp}_{2,3+}(@ 63.66 \mathrm{eV})$ doubly-excited states at various time delays (the negative value corresponds to VIS first). For negative time delays the VIS laser pulse has no effect on the system, thus the typical asymmetric Fano line shape is observed. For positive time delays the VIS laser pulse couples the SXR-excited states and thus alters the dipole emission imprinted on the SXR spectra. The effect is most visible on the $\mathrm{sp}_{2,3+}$ state, which completely changes its shape (including inversion) on a few 100 attosecond timescale. $b, c)$ Modulation of the transient-absorption signal as a function of photon energy and time delay for the experimental (b) and simulated (c) results. Both the $2 \mathrm{~s} 2 \mathrm{p}$ as well as the $\mathrm{sp}_{2,3+}$ resonance exhibit a fast modulation pattern, which is reproduced by the model, taking into account the relevant autoionizing states and their laser-induced coupling. The VIS intensity was $3 \times 10^{12} \mathrm{~W} / \mathrm{cm}^{2}$.

\section{References}

1. J. Mauritsson, T. Remetter, M. Swoboda, K. Klünder, A. L'Huillier, K. J. Schafer, O. Ghafur, F. Kelkensberg, W. Siu, P. Johnsson, M. J. J. Vrakking, I. Znakovskaya, T. Uphues, S. Zherebtsov, M. F. Kling, F. Lépine, E. Benedetti, F. Ferrari, G. Sansone, M. Nisoli, Phys. Rev. Lett. 105, 053001 (2010).

2. M. Holler, F. Schapper, L. Gallmann, U. Keller, Phys. Rev. Lett. 106, 123601 (2011).

3. L. Argenti, E. Lindroth, Phys. Rev. Lett. 105, 053002 (2010).

4. C. Ott, A. Kaldun, P. Raith, K. Meyer, M. Laux, Y. Zhang, S. Hagstotz, T. Ding, R. Heck, T. Pfeifer (submitted 2012).

5. E. Goulielmakis, Z.-H. Loh, A. Wirth, R. Santra, N. Rohringer, V. S. Yakovlev, S. Zherebtsov, T. Pfeifer, A. M. Azzeer, M. F. Kling, S. R. Leone, F. Krausz, Nature 466, 739-743 (2010).

6. S. Gilbertson, M. Chini, X. Feng, S. Khan, Y. Wu, Z. Chang, Phys. Rev. Lett. 105, 263003 (2010).

7. Z.-H. Loh, C. H. Greene, and S. R. Leone, Chem. Phys. 350, 7-13 (2008). 